\title{
A new dermocosmetic containing retinaldehyde, delta-tocopherol glucoside and glycylglycine oleamide for managing naturally aged skin: results from in vitro to clinical studies
}

\author{
This article was published in the following Dove Press journal: \\ Clinical, Cosmetic and Investigational Dermatology \\ 2 February 2017 \\ Number of times this article has been viewed
}

\author{
Céline Rouvrais ${ }^{1, *}$ \\ Daniel Bacqueville ${ }^{2, *}$ \\ Patrick Bogdanowicz ${ }^{2, *}$ \\ Marie-José Haure ${ }^{2}$ \\ Laure Duprat ${ }^{2}$ \\ Christine Coutanceau ${ }^{3}$ \\ Nathalie Castex-Rizzi ${ }^{2}$ \\ Hélène Duplan ${ }^{2}$ \\ Valérie Mengeaud' \\ Sandrine Bessou-Touya ${ }^{2}$ \\ 'Clinical Skin Research Center, \\ ${ }^{2}$ Department of Pharmacology, Pierre \\ Fabre Dermo-Cosmétique, Toulouse, \\ ${ }^{3}$ Laboratoire Dermatologique Avène, \\ Lavaur, France
}

*These authors contributed equally to this work

Correspondence: Daniel Bacqueville Department of Pharmacology, Pierre Fabre Dermo-Cosmétique, Avenue

Hubert Curien, BP I3562, 31035

Toulouse Cedex I, France

Tel +33534506427

$\mathrm{Fax}+33534503424$

Email daniel.bacqueville@pierre-fabre.com
Introduction: Natural aging of skin tissues, the addition of the cumulative action of the time and radiation exposure result in skin atrophy, wrinkles and degeneration of the extracellular matrix (ECM). The aim of the study was to investigate the beneficial effect of a combination containing retinaldehyde (RAL), delta-tocopherol glucoside (delta-TC) and glycylglycine oleamide (GGO) and of a dermocosmetic containing the combination.

Materials and methods: The protective effect of the combination was assessed through in vitro gene expression of ultraviolet (UV)-irradiated fibroblasts. A skin aging assay using UV light on ex vivo skin samples and a clinical study conducted in 36 women aged from 35 to 55 years with a minimum of level 4 to a maximum of level 6 on the crow's feet photoscale assessed the antiaging effect of the dermocosmetic.

Results: When added to UV-irradiated fibroblasts, the combination substantially improved the ECM in activating the elastin fiber production (fibrillin 2, fibulin 1 and 5 and lysyl oxidase-like 2) as well as that of proteins involved in the cellular ECM interactions (integrin $\beta 1$, paxillin and actin a2). An ex vivo photodamaged human skin model showed that the dermocosmetic formulation containing the combination of the active ingredients protected the elastic network against UV-induced alterations including both elastin and fibrillin-rich fibers in the dermis. A daily application of the dermocosmetic for 2 months on naturally aged skin resulted in a statistically significant improvement $(p<0.05)$ of visible signs of aging comprising crow's feet, wrinkles and periocular fine lines. Finally, the formulation was well tolerated.

Conclusion: The dermocosmetic containing RAL, delta-TC and GGO provides a substantial benefit in the daily care of naturally aged skin in women aged 35-55 years.

Keywords: glycylglycine oleamide, delta-tocopherol glucoside, retinaldehyde, preclinical aged skin model, statistics, formulation

\section{Introduction}

Aging of the skin is a more and more important concern in the daily skin care of women in their 30s.

Aging of organic tissues and especially of the skin is a natural and intricate biological process consisting of 2 types: intrinsic or chronological aging which is an inevitable process and photoaging which involves the premature aging of skin due to cumulative exposure to ultraviolet (UV) radiation as well as of the amount of melanin in the skin. ${ }^{1,2}$ Aged dermis constitutively expresses elevated levels of several matrix metalloproteinases (MMPs) and reduced production of type I collagen, which likely 
leads to chronic, progressive degradation of the dermal collagenous extracellular matrix (ECM) and loss of collagen. This aberrant collagen homeostasis largely results from altered fibroblast function due to long-term consequences of fragmented dermal collagen microenvironment in aged skin. ${ }^{3,4}$

Skin aging signs can be classified into 4 main categories: wrinkles/fine lines, lack of firmness of cutaneous tissues (ptosis), vascular disorders and pigmentation heterogeneities. ${ }^{5-7}$ Wrinkles and fine lines, so-called crow's feet, appear especially in sun-exposed areas of the skin, such as the face in the periocular zones, neck, upper chest, hands and forearms; these are the zones of the body where the changes are most visible..$^{8,9}$

Retinoids were introduced more than 2 decades ago to manage skin aging. ${ }^{1,10,11}$ The retinoid family includes retinol (vitamin A, retinol) and its natural derivatives retinaldehyde (RAL), retinoic acid and retinyl esters, as well as a large number of synthetic derivatives. ${ }^{10}$ Vitamin A cannot be synthesized by the body and needs to be supplied exogenously. It is an important constituent of the epidermis, where it is involved in the epidermal turnover. A deficiency may be caused by exposure to sunlight or any UV source, an oxidative stress and by chronological aging. As a consequence, any treatment aimed at increasing epidermal vitamin A exerts protective effects against these deleterious conditions. ${ }^{12}$ Retinaldehyde or retinol has been shown to prevent epidermal Vitamin A deficiency. It may be combined with other compounds with complementary actions against aging, nutritional deficiency and cancer such as antioxidants to potentiate their beneficial effects in the skin. ${ }^{12}$

Free radicals or oxidants have long been studied as contributors to aging and disease processes. Their endogenous production from cellular metabolism and exogenous sources from UV radiation and pollution damages the skin at the cellular and tissue levels. ${ }^{13}$ Although the body has a defense system to prevent radical damage, this innate system can be overwhelmed leading to a state of oxidative stress or immunosuppression that can even trigger carcinogenesis. ${ }^{12,14}$

Vitamin E or alpha-tocopherol has been used for $>50$ years in experimental and clinical dermatology. ${ }^{15,16}$ Delta-tocopherol glucoside (Pretocopheryl ${ }^{\circledR}$ [Pierre Fabre Médicament, Gaillac, France], hereafter delta-TC) is a precursor of vitamin $\mathrm{E}$, well known for its antioxidation potential and associated with a better stability and gradual delivery of free tocopherol in the skin. ${ }^{17}$ In a clinical study, an association of RAL and delta-TC significantly decreased the number of $\mathrm{UV}$-generated free radicals, resulting in a superior restoration of laminin, elastic fiber and collagen network and increased the collagen synthesis more than RAL alone. ${ }^{18}$

Glycation is an aging reaction of naturally occurring sugars with dermal proteins, during which the natural suppleness of the dermis is affected by the formation of bridges between proteins and sugars. ${ }^{19,20}$ The presence of glycated collagen can be observed as early as at the age of 20 years. It accumulates with a yearly rate of $\sim 3.7 \%$ reaching a $30-50 \%$ increase at 80 years of age. ${ }^{21-23}$

Glycylglycine oleamide (GGO) is a small amphiphilic molecule that protects the connective tissue of the skin from glycation and from elastosis of fibrilline-1. Both are considered potential targets of antiaging products as demonstrated recently through in vitro and ex vivo studies on GGO. ${ }^{24}$

The present study reports results for a combination containing RAL, delta-TC and GGO known for their individual activities, from in vitro to ex vivo investigations and from a clinical study conducted in women with mild to moderate naturally aged skin.

\section{Materials and methods In vitro assay on the impact of the combination on the gene expression of irradiated fibroblasts}

Normal human dermal fibroblasts (NHDF) were obtained from 4 different donors, having provided prior to sampling written donor permission according to the French regulations on donor rights. Cell samples were cultured at $37^{\circ} \mathrm{C}$, and $\mathrm{CO}_{2}$ at $5 \%$, in a DMEM enriched with L-glutamine $2 \mathrm{mM}$, penicillin $50 \mathrm{U} / \mathrm{mL}$, streptomycin $50 \mu \mathrm{g} / \mathrm{mL}$ and SVF $10 \%$.

Fibroblasts were seeded on 6-well plates and cultured in the media for 24 hours. The culture media were replaced by the test media consisting of DMEM enriched with SVF $1 \%$, and the cells were preincubated for 24 hours. After the preincubation period, the media were replaced by the irradiation media consisting of Earle's Balanced Salt Solution (EBSS) enriched with $\mathrm{CaCl}_{2} 0.264 \mathrm{~g} / \mathrm{L}$ and $\mathrm{MgSO}_{4} 0.2 \mathrm{~g} / \mathrm{L}$. Samples were irradiated or not (nonirradiated control) using ultraviolet A (UVA) at $20 \mathrm{~J} / \mathrm{cm}^{2}$. After irradiation, the media were replaced by the test media, containing or not (irradiated or control, respectively) the combination of RAL $1 \mu \mathrm{M}$, deltaTC $10 \mu \mathrm{M}$ and GGO $1 \mu \mathrm{M}$. Cells were incubated for 48 hours.

To assess the reverse transcription, the total RNA of each sample was extracted using as indicated the TriPure Isolation Reagent $^{\circledR}$ (Sigma-Aldrich, Saint-Louis, MO, USA). RNA was quantified using spectrophotometry (Nanovue; GE Healthcare, Little Chalfont, UK), and its quality was 
evaluated using capillary electrophoresis (Bioanalyzer 2100; Agilent, Santa Clara, CA, USA). Complementary DNA (cDNA) was synthesized by reverse transcription from $1 \mu \mathrm{g}$ total RNA in the presence of oligo (dT) and the enzyme Superscript II (Thermo Fisher Scientific, Waltham, MA, USA). The RNA quantity was adjusted by water dilution for performing polymerase chain reactions (PCRs) by quantitative PCR (Light Cycler; Roche Molecular Systems Inc., Pleasanton, CA, USA) according to the supplier's recommendations. The reactive mix $(10 \mu \mathrm{L}$ final $)$ of each sample contained 2.5 $\mu \mathrm{L}$ of cDNA, gene probes, DNA polymerase, SYBR Green I and $\mathrm{MgCl}_{2}$.

Quantification data were analyzed with the delta-delta $\mathrm{Ct}$ method by comparison with control untreated and treated cells. Assays for 3 housekeeping genes were included in the studies for normalization of the data ( $\beta$-glucuronidase, importin 8 and ubiquitin C). Results are expressed in "fold of increase", as mean \pm SD of 4 independent experiments. The Student's $t$-test was performed to determine the statistical significance with a threshold of $p<0.05$.

\section{Ex vivo and clinical testing of the combination in a dermocosmetic Composition of the dermocosmetic formulations}

A preformulation was first prepared to test the efficacy of the combination of RAL $0.05 \%$, delta-TC $0.05 \%$ and GGO $0.1 \%$ in an ex vivo human skin model. This formulation was evaluated versus a placebo and was prepared by using Avène thermal spring water and routine excipients such as caprylic/ capric triglyceride, mineral oil and glyceryl stearate.

A dermocosmetic was also formulated with a combination of RAL $0.1 \%$, delta-TC $0.1 \%$ and GGO $0.1 \%$, and its antiaging properties were evaluated both in the ex vivo human skin model and in a clinical study. International Nomenclature of Cosmetic Ingredients nomenclature was Avène thermal spring water, triethylhexanoin, cyclopentasiloxane, cetearyl alcohol, cyclohexasiloxane, ceteareth-20, polymethyl methacrylate, ceteareth-33, caprylic/capric triglyceride, ammonium acryloyl dimethyltaurate/VP copolymer, benzoic acid, BHT, caramel, disodium EDTA, mica, Oenothera biennis (evening primrose) oil, oleoyl dipeptide-15, oleoyl tetrapeptide-31, phenoxyethanol, red 33, retinal, silica, sodium hydroxide, titanium dioxide, tocopherol and tocopheryl glucoside (Ysthéal, Avène, Pierre Fabre Dermo Cosmétique, Lavaur, France).

\section{Evaluation in human skin organ culture using an ex vivo skin aging assay}

Assays were performed using fresh human skin obtained from abdominoplasty specimens from patients having provided, prior to the intervention, written donor consent according to the French legal requirements on donor rights. Explants were excised and seeded in polycarbonate inserts of a 6-well plate containing a survival medium based on DMEM supplemented with antibiotics and an antifungal agent. ${ }^{25}$ This ex vivo organ culture system maintained the skin at the air-liquid interface and fed dermis and epidermis through nutrient diffusion across the insert. Human skin was incubated at $37^{\circ} \mathrm{C}$ in a humid atmosphere with $5 \% \mathrm{CO}_{2}$. Tissues were exposed to a single UVA dose of $12 \mathrm{~J} / \mathrm{cm}^{2}$ using a Biosun Vilber Lourmat (Vilber, Eberhardzell, Germany) simulator at $365 \mathrm{~nm}$ wavelength. Following UVA exposure, the preformulation (or the placebo) and the dermocosmetic were applied topically on the skin surface at $10 \mathrm{mg} / \mathrm{cm}^{2}$, immediately. After 24 hours of incubation, the same dose was applied again. Skin samples were analyzed 24 hours after the last application, resulting in a total application duration of 48 hours.

Skin was then harvested for fibrillin analyses and elastin expression using double immunostaining from $6 \mu \mathrm{m}$ cryosections. Samples were fixed in acetone, saturated in TBS/ Triton X-100/goat serum and stained overnight at $4{ }^{\circ} \mathrm{C}$ with primary antibodies directed against fibrillin (1/100) and elastin (1/500). Revelation was achieved using secondary antibodies coupled to Alexa Fluor ${ }^{\circledR} 488$ and 594 (1/500), respectively. Slides were mounted in Prolong medium with nuclear dye DAPI. Fluorescent signals were observed by using a confocal microscope Nikon $\mathrm{Al}^{+}$. Histological staining of elastin fibers was performed using Orcein according to the standard procedures.

\section{Clinical study}

A noncomparative, monocentric, open-label 2-month study was conducted in women with chronologically aged skin to assess the potential of the dermocosmetic to attenuate wrinkles (crow's feet) and periocular fine lines.

In France, regulation does not require approval from an ethics committee/international review board (IRB) when a product is a cosmetic used on healthy volunteers and assesses with a noninvasive technology. This clinical single-center study was carried out at the Centre de Recherche sur la Peau Pierre Fabre (CRP), Toulouse, France, according to the ethical principles of the Declaration of Helsinki and the guidelines for Good Clinical Practices (CPMP/ICH/135/95). Subjects were recruited from a panel of healthy volunteers identified by using a database reported to the French Data Protection Authority (Commission Nationale de l'Informatique et des Libertés). All subjects provided written informed consent prior to application of the dermocosmetic 
A

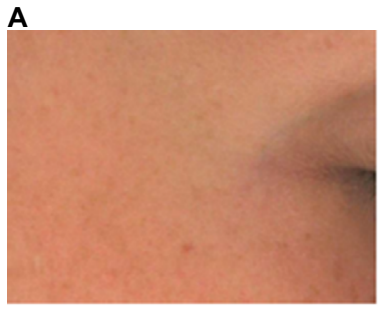

Grade 0

D

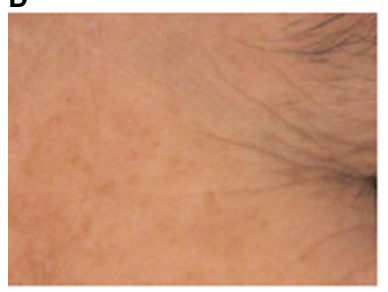

Grade 6

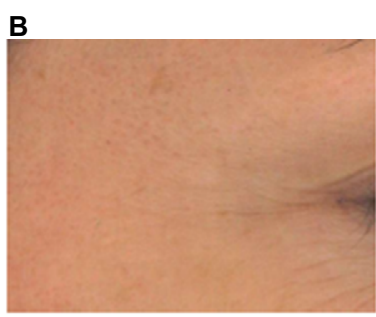

Grade 2

E

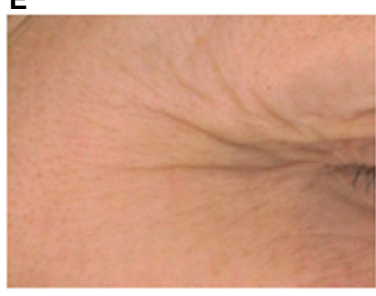

Grade 8

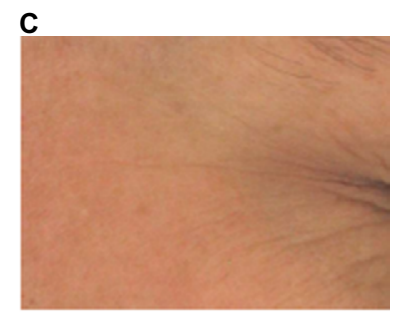

Grade 4

$\mathbf{F}$

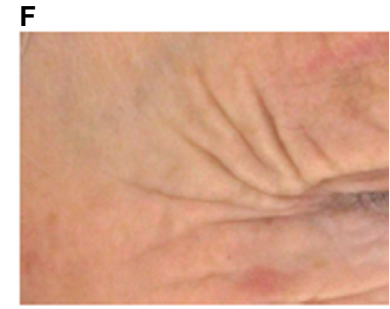

Grade 10

Figure I Pierre Fabre Dermo-Cosmetics Crow's feet wrinkle photo scale.

Notes: The photo scale was graded according to wrinkle appearance: (A) Grade 0; (B) Grade 2; (C) Grade 4 (D) Grade 6; (E) Grade 8; and (F) Grade I0.

and a copyright transfer agreement for photographs. Suitable female subjects had to be of age 35-55 years and had to have a phototype I to III on the Fitzpatrick scale. Each subject had to have a minimum level of 4 up to a maximum of level 6 on the Pierre Fabre Dermo-Cosmetic crow's feet photo scale (Figure 1).

Participating women were not allowed to use facial or oral antiaging products during the study other than the dermocosmetic to be tested. No topical or oral retinoids were used within the 4 or 6 months, respectively, preceding the study participation.

The dermocosmetic was applied daily in the evening for 2 months. Subjects received sunscreen products with a $\mathrm{SPF}^{+} 0^{+}$(Laboratoire Dermatologique Avène, Lavaur, France) to be applied on the face each sun exposure and to be reapplied as necessary.

Skin surface changes in crow's feet and in periocular areas were analyzed using profilometry on skin negative replica; the length of crow's feet wrinkles was quantified by image analysis. Local tolerance was assessed throughout the study.

Statistical analyses were descriptive for baseline demographics. Statistical analyses of efficacy parameters on the face were performed using the Student's $t$-test or Wilcoxon test.

\section{Results}

\section{In vitro assay on the impact of the combination on the gene expression of irradiated fibroblasts}

After 48 hours of UVA irradiation at $20 \mathrm{~J} / \mathrm{cm}^{2}$, gene expression coding for proteins involved in the assembling of elastin fibers was reduced compared to the control level: lysyl oxidase-like 2 (LOXL2: 0.67), fibulin 1 (FBLN1: 0.76), fibulin 5 (FBLN5: 0.61) and fibrillin 2 (FBN2: 0.60). Expression of genes implicated in the cell-ECM interaction, integrin $\beta 1$ (ITGB1: 0.69) and paxillin (PXN: 0.61) as well as the cytoskeleton, actin alpha 2 smooth muscle aorta (ACTA2: 0.54) was inhibited.

When added during the 48 hours following irradiation, the combination restored and improved the expression level of genes coding for the formation of elastin fibers, the cell-ECM interaction and the cytoskeleton, reaching almost or exceeding the level of the control samples: LOXL2: 1.22, FBLN1: 1.57, FBLN5: 1.03, FBN2: 1.00, ITGB1: 1.15, PXN: 1.66 and ACTA2: 0.99. Results are presented in Table 1; all were statistically significant $(p<0.05)$.

\section{Evaluation of the combination on elastic network in human skin organ culture using an ex vivo skin aging assay}

The ex vivo photodamaged human skin model confirmed that UVA impairs the cutaneous elastic system altering both elastin and fibrillin-rich fibers in the dermis (Figure 2, red and green coloration, respectively). The elastic network was strongly disorganized following UVA irradiation, especially in the papillary dermis close to the dermoepidermal junction (dashed line). The fibrillin-rich fibers were clearly cut and shorter under the epidermis, while elastin fibers were destroyed and accumulated in the deeper dermis. UVA irradiation of the skin explants damaged the fibrillin expression and elastin in the dermis and caused an important disruption of the network of elastic fibers with multiple breaks. 
Table I Impact of the combination RAL, delta-TC and GGO on the gene expression of irradiated fibroblasts

\begin{tabular}{|c|c|c|c|c|c|}
\hline Gene code & Gene name & Control & $\begin{array}{l}\text { Mean ratio } \\
\text { UV/control } \\
\text { (SD) }\end{array}$ & $\begin{array}{l}\text { Mean ratio } \\
\text { (UV + combination)/UV } \\
\text { (SD) }\end{array}$ & $\begin{array}{l}p \text {-Value } \\
(U V+\text { combination) } \\
\text { versus UV }\end{array}$ \\
\hline \multicolumn{6}{|c|}{ Elastic fiber formation } \\
\hline LOXL2 & Lysyl oxidase-like 2 & 1 & $0.67 \pm 0.18$ & $1.22 \pm 0.18$ & 0.026 \\
\hline FBLNI & Fibulin I & 1 & $0.76 \pm 0.22$ & $1.57 \pm 0.11$ & 0.007 \\
\hline FBLN5 & Fibulin 5 & 1 & $0.61 \pm 0.10$ & $1.03 \pm 0.23$ & 0.030 \\
\hline FBN2 & Fibrillin 2 & 1 & $0.60 \pm 0.08$ & $1.00 \pm 0.21$ & 0.031 \\
\hline \multicolumn{6}{|c|}{ ECM-receptor interaction } \\
\hline ITGBI & Integrin, $\beta$ I & 1 & $0.69 \pm 0.08$ & $1.15 \pm 0.22$ & 0.011 \\
\hline$P X N$ & Paxillin & 1 & $0.61 \pm 0.17$ & $1.66 \pm 0.75$ & 0.044 \\
\hline \multicolumn{6}{|l|}{ Cytoskeleton } \\
\hline ACTA2 & $\begin{array}{l}\text { Actin, alpha2 smooth } \\
\text { muscle aorta }\end{array}$ & I & $0.54 \pm 0.14$ & $0.99 \pm 0.31$ & 0.038 \\
\hline
\end{tabular}

Abbreviations: RAL, retinaldehyde; delta-TC, delta-tocopherol glucoside; GGO, glycylglycine oleamide; UV, ultraviolet.

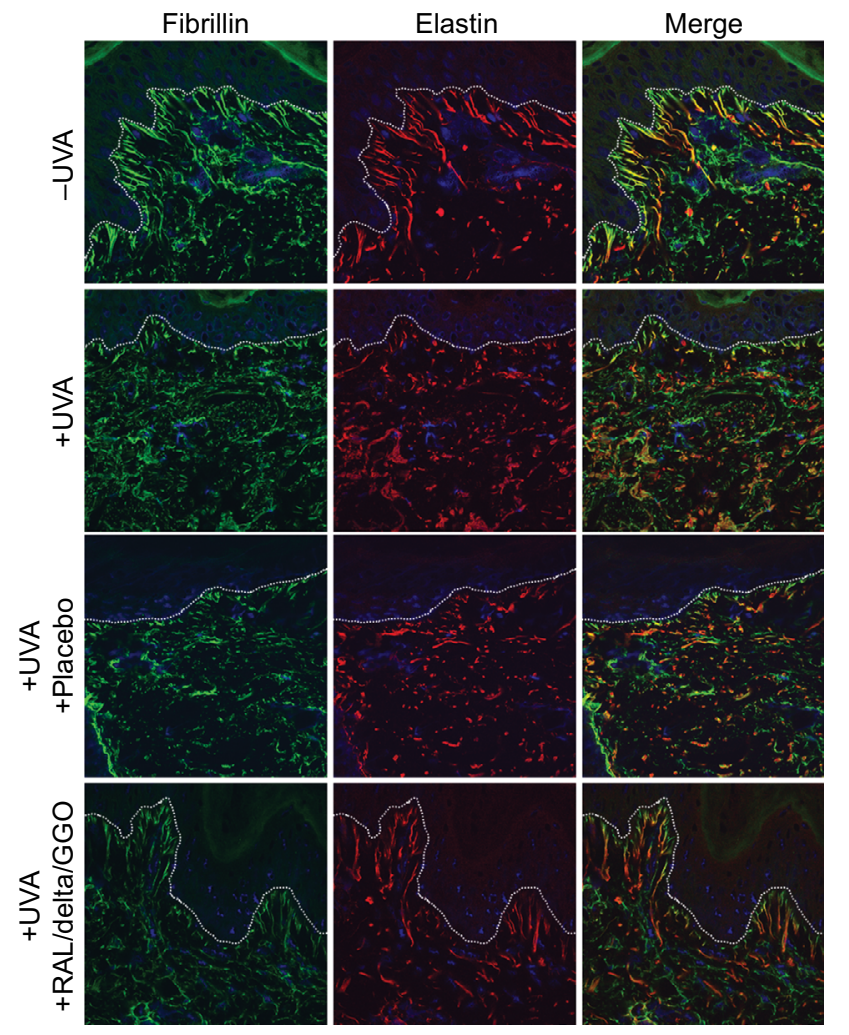

Figure 2 Not irradiated (-UVA), UVA-irradiated (+UVA) and UVA-irradiated human skin explants treated with a placebo or a preformulation containing a combination of RAL/delta-TC/GGO assessed by using immunohistochemistry and confocal microscopy.

Notes: UVA induced a strong alteration of the elastic network, especially in the papillary dermis close to the dermoepidermal junction (dashed line). The fibrillinrich fibers and elastin were visualized in green and red colors, respectively. Blue color denotes cell nuclei. Merge showed colocation of both proteins in the skin. The preformulation protected the dermis against UVA damage, whereas a placebo was not effective to prevent elastin network degradation. Field of view: $215 \times 215 \mu \mathrm{m}$. Abbreviations: UVA, ultraviolet A; RAL, retinaldehyde; delta-TC, delta-tocopherol glucoside; GGO, glycylglycine oleamide.

After 2 topical applications of the preformulation containing the 3 active ingredients within 48 hours after irradiation on the skin explants, immunostaining results of fibrillin and elastin showed that the structure of the elastic network of

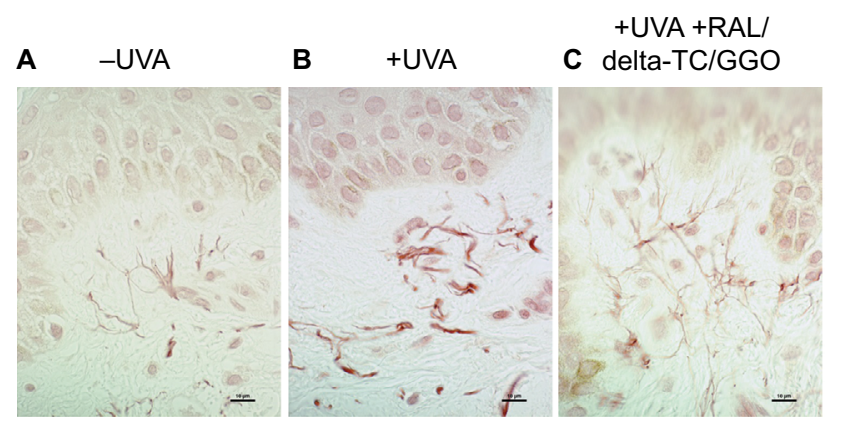

Figure 3 Ex vivo histology using Orcein staining of skin explants (A) before (-UVA), (B) after UVA (+UVA) and (C) after UVA and a preformulation containing a combination of RAL/delta-TC/GGO.

Note: Scale bar $=10 \mu \mathrm{m}$.

Abbreviations: UVA, ultraviolet A; RAL, retinaldehyde; delta-TC, delta-tocopherol glucoside; GGO, glycylglycine oleamide.

both irradiated and treated skin samples was almost identical to that of nonirradiated control samples (Figure 2). Moreover, the placebo control was not effective to protect the skin against UVA-induced elastin alterations. Results were confirmed by the detection of elastin fibers after histological staining using Orcein (Figure 3).

\section{Preclinical and clinical studies}

Supported by these results, a combination of RAL $0.1 \%$, delta-TC $0.1 \%$ and GGO $0.1 \%$ was formulated as a dermocosmetic. In parallel to a clinical study, its effect on the elastic network was assessed ex vivo following topical application, as previously shown with the preformulation. The ex vivo confocal analyses revealed that the dermocosmetic prevented both fibrillin and elastin degradation in response to UVA and may be used as an antiaging formulation (Figure 4).

A clinical study was performed to confirm its efficacy in naturally aged skin in women. 


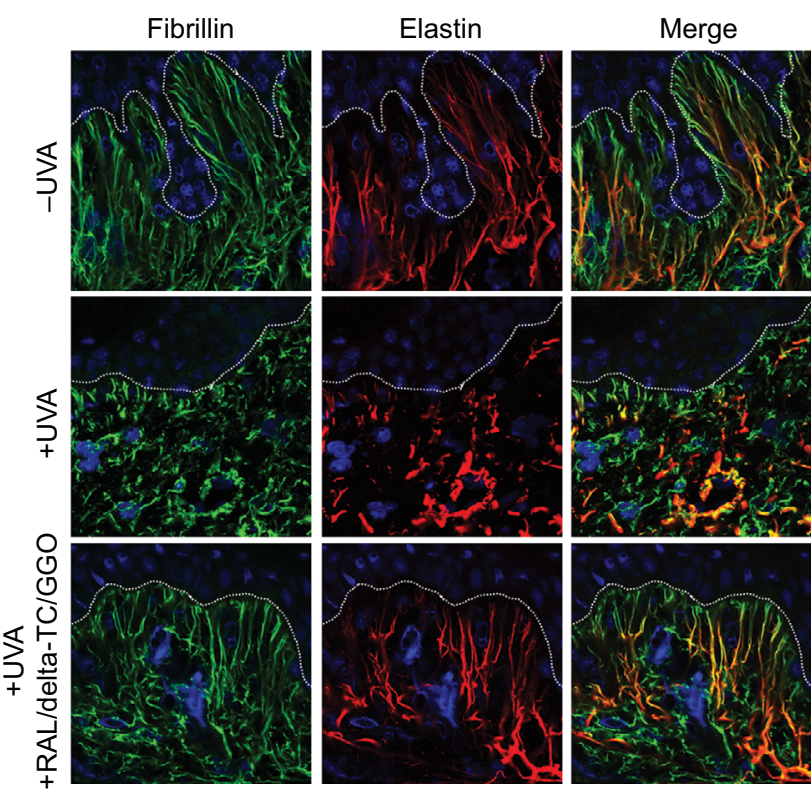

Figure 4 Not irradiated (-UVA), UVA-irradiated (+UVA) and UVA-irradiated human skin explants treated with a dermocosmetic formulation containing a combination of RAL/delta-TC/GGO assessed by using immunohistochemistry and confocal microscopy.

Notes: A dermocosmetic product containing a combination of RAL $0.1 \% /$ deltaTC $0.1 \% / G G O 0.1 \%$ was formulated and was able to prevent UVA-induced elastin network alterations in the dermis. Dashed line indicates the dermoepidermal junction. Fibrillin-rich fibers and elastin are visualized in green and red colors, respectively. Blue color denotes cell nuclei. Merge showed colocation of both proteins in the skin. Field of view: $123 \times 123 \mu \mathrm{m}$.

Abbreviations: UVA, ultraviolet A; RAL, retinaldehyde; delta-TC, delta-tocopherol glucoside; GGO, glycylglycine oleamide.

\section{Demographic and baseline data}

A total of 36 women meeting the inclusion criteria participated in the clinical study; 1 subject withdrew from the study due to a local adverse reaction (moderate facial erythema) after 1 month of application and a second withdrew between visits of Month 1 and 2 due to a pregnancy followed by an abortion; 34 women completed the study.
The mean age was 46.60 years with a minimum of 38 and a maximum of 56 years.

\section{Efficacy}

\section{Cutaneous relief by profilometric measurements}

After 2 months of application, the peak-valley difference relative to the total surface $(154.56 \pm 37.45 \mu \mathrm{m})$ for crow's feet had significantly improved $(p=0.0108)$ by $5.4 \%$ compared to baseline $(163.68 \pm 37.53 \mu \mathrm{m})$ with a significant onset as early as Month $1(4.2 \%, p=0.0375)$. The volume under the mean level had not significantly improved after 2 months.

After 2 months of application, the peak-valley difference relative to the total surface $(115 \pm 26.38 \mu \mathrm{m})$ for fine lines in the subocular zone had significantly improved by $11.16 \%(p<0.0014)$ compared to baseline $(139.81 \pm 60.98 \mu \mathrm{m})$. The volume under the mean level after 2 months $\left(-0.098 \pm 0.067 \mathrm{~mm}^{3}\right)$ had significantly improvement by $26.10 \%(p=0.0276)$ compared to baseline $\left(-0.144 \pm 0.097 \mathrm{~mm}^{3}\right)$.

\section{Length of periocular wrinkles}

After 2 months of application, the length of periocular wrinkles $(2574.97 \pm 1185.31$ pixels $)$ had significantly improved by $15.5 \%(p=0.033)$ compared to baseline (3047.82 \pm 1486.15 pixels).

Percent improvements for all assessed parameters are depicted in Figure 5.

\section{Tolerance}

After 2 months of daily application, the tolerance of the dermocosmetic containing the combination assessed by the investigators was good to very good in $80 \%$ of the volunteers.

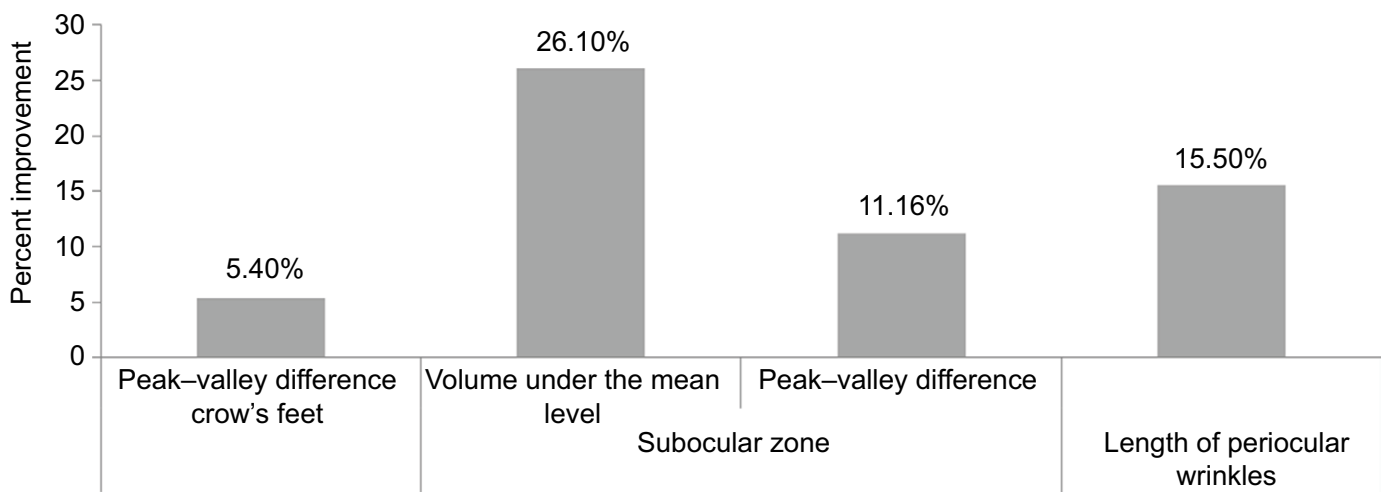

Figure 5 Improvement of skin aging parameters using specific devices after 2 months of daily application of the combination of a dermocosmetic formulation containing a combination of RAL/delta-TC/GGO.

Notes: Skin surface changes in crow's feet and in subocular area wrinkles were analyzed by profilometric technique on skin negative replicas. The length of crow's feet wrinkles was quantified by image analysis on photographs. Changes from baseline for all assessed parameters were statistically significant ( $p<0.05$ ).

Abbreviations: RAL, retinaldehyde; delta-TC, delta-tocopherol glucoside; GGO, glycylglycine oleamide. 


\section{Discussion and conclusion}

The aim of the present study was to assess the antiaging effects of a combination of RAL, delta-TC and GGO in several cutaneous models. UVA-irradiated dermal fibroblasts showed that these active ingredients improve gene expression in vitro, whereas a skin organ culture revealed the efficacy of a preformulated combination containing RAL/delta-TC/GGO to protect the cutaneous elastic network, after topical application. Furthermore, a clinical study was carried out testing a combination of RAL $0.1 \%$, delta-TC $0.1 \%$ and GGO $0.1 \%$ formulated as a dermocosmetic to prove its antiaging properties. Results showed the benefit of the active ingredients combination after 2-month topical application in naturally aged skin in women. Finally, the ex vivo human skin model also supported the protective effects of the dermocosmetic RAL/delta-TC/GGO on the elastic network.

Photodamage is associated with elastic network disorganization potentially related to solar elastosis, a clinical feature associated with photoaging and excessive sun exposure. Our ex vivo skin model confirmed that UVA induces alteration of the skin structure demonstrated by Boisnic et al. ${ }^{26}$ When adding, during the 48 hours following UVA irradiation, the tested combination, a restoration and improvement of the expression level of genes coding for the formation of elastin fibers, the cell-ECM interaction and the cytoskeleton, such as LOXL2, FBLN1, FBLN5, FBN2, ITGB1, PXN and ACTA2 were observed paralleling findings made earlier when RAL, GGO and delta-TC were tested individually or after application of a combination of RAL and delta-TC in ex vivo skin samples. ${ }^{18,24,26,27}$ The presently tested combination of 3 actives thus effectively protected skin tissues from UVA radiation and preserved the structure of both elastin and fibrillin-rich fibers preventing UVA-induced photoaging and preserving the elastic system organization. It is of note that a placebo preformulation was not efficient to protect the dermis against UVA, confirming the topical efficacy of the combined active ingredients.

The clinical study conducted in the target population confirmed ex vivo findings. A 2-month daily application of a dermocosmetic containing RAL $0.1 \%$, delta-TC $0.1 \%$ and GGO $0.1 \%$ significantly improved $(p<0.05)$ visible signs of naturally aged skin, comprising periocular fine lines and crow's feet. The combination formulated in a dermocosmetic effectively protected the skin from UVA damages and repaired visible signs of skin aging. Local tolerance of the dermocosmetic was good and similar to that observed with topical retinoids and topical products containing retinoids..$^{28,29}$ The ex vivo observations showed that the dermocosmetic efficiently preserves the elastic network in the skin and therefore support that the visible antiaging effects of the formulation in women are related to a benefit of the active ingredients on the elastic network, as suggested in previous studies. ${ }^{24,26}$

We concede that the chosen noncomparative design of the reported clinical study may be considered a limitation as it does not allow assessing objectively the efficacy of the combination in a dermocosmetic formulation when compared to retinol or to other comparable antiaging products. Indeed to date, management of skin aging is no longer limited to interventional procedures or the use of tretinoin alone, the gold standard of topical management of aged skin, ${ }^{30}$ as more and more dermocosmetics are available limiting damages caused by exo- and endogenous skin aging triggers. However, the aim of this study was not to show superiority over or noninferiority compared to other currently marketed topical antiaging products, but to propose a new noninvasive and well-tolerated alternative, allowing limiting efficiently, clinically visible signs of skin aging as early as the 30 's.

In conclusion, we consider that the tested dermocosmetic containing RAL, delta-TC and GGO associated with Avène thermal spring water appears to be effective in treating the natural signs of aging.

\section{Acknowledgments}

The reported studies were funded by Pierre Fabre DermoCosmétique, France and Laboratoire Dermatologique Avène. Karl Patrick Göritz, SMWS, France provided writing support.

\section{Disclosure}

All authors are employees of Pierre Fabre Dermo-Cosmétique, France. The authors report no other conflicts of interest in this work.

\section{References}

1. Mukherjee S, Date A, Patravale V, Korting HC, Roeder A, Weindl G. Retinoids in the treatment of skin aging: an overview of clinical efficacy and safety. Clin Interv Aging. 2006;1(4):327-348.

2. Uitto J. The role of elastin and collagen in cutaneous aging: intrinsic aging versus photoexposure. J Drugs Dermatol. 2008;7(2 Suppl) s12-s16

3. Quan T, Little E, Quan H, Qin Z, Voorhees JJ, Fisher GJ. Elevated matrix metalloproteinases and collagen fragmentation in photodamaged human skin: impact of altered extracellular matrix microenvironment on dermal fibroblast function. J Invest Dermatol. 2013;133(5):1362-1366.

4. Chung JH, Seo JY, Choi HR, et al. Modulation of skin collagen metabolism in aged and photoaged human skin in vivo. $J$ Invest Dermatol. 2001;117(5):1218-1224.

5. Flament F, Bazin R, Laquieze S, Rubert V, Simonpietri E, Piot B. Effect of the sun on visible clinical signs of aging in Caucasian skin. Clin Cosmet Investig Dermatol. 2013;6:221-232.

6. Gilchrest BA. Skin aging and photoaging. Dermatol Nurs. 1990;2(2):79-82 
7. Yaar M, Eller MS, Gilchrest BA. Fifty years of skin aging. J Investig Dermatol Symp Proc. 2002;7(1):51-58.

8. Helfrich YR, Sachs DL, Voorhees JJ. Overview of skin aging and photoaging. Dermatol Nurs. 2008;20(3):177-183. quiz 184.

9. Gordon JR, Brieva JC. Images in clinical medicine. Unilateral dermatoheliosis. N Engl J Med. 2012;366(16):e25.

10. Ramos-e-Silva M, Hexsel DM, Rutowitsch MS, Zechmeister M. Hydroxy acids and retinoids in cosmetics. Clin Dermatol. 2001; 19(4):460-466.

11. Kligman LH, Duo CH, Kligman AM. Topical retinoic acid enhances the repair of ultraviolet damaged dermal connective tissue. Connect Tissue Res. 1984;12(2):139-150.

12. Sorg O, Saurat JH. Topical retinoids in skin ageing: a focused update with reference to sun-induced epidermal vitamin A deficiency. Dermatology. 2014;228(4):314-325.

13. Podda M, Traber MG, Weber C, Yan LJ, Packer L. UV-irradiation depletes antioxidants and causes oxidative damage in a model of human skin. Free Radic Biol Med. 1998;24(1):55-65.

14. Chen L, Hu JY, Wang SQ. The role of antioxidants in photoprotection: a critical review. J Am Acad Dermatol. 2012;67(5):1013-1024.

15. Thiele JJ, Ekanayake-Mudiyanselage S. Vitamin E in human skin: organ-specific physiology and considerations for its use in dermatology. Mol Aspects Med. 2007;28(5-6):646-667.

16. Gensler HL, Magdaleno M. Topical vitamin E inhibition of immunosuppression and tumorigenesis induced by ultraviolet irradiation. Nutr Cancer. 1991;15(2):97-106.

17. Mavon A, Raufast V, Redoules D. Skin absorption and metabolism of a new vitamin E prodrug, delta-tocopherol-glucoside: in vitro evaluation in human skin models. J Control Release. 2004;100(2):221-231.

18. Boisnic S, Branchet-Gumila MC, Nocera T. Comparative study of the anti-aging effect of retinaldehyde alone or associated with pretocopheryl in a surviving human skin model submitted to ultraviolet A and B irradiation. Int J Tissue React. 2005;27(3):91-99.

19. Gasser P, Arnold F, Peno-Mazzarino L, et al. Glycation induction and antiglycation activity of skin care ingredients on living human skin explants. Int J Cosmet Sci. 2011;33(4):366-370.
20. DeGroot J. The AGE of the matrix: chemistry, consequence and cure. Curr Opin Pharmacol. 2004;4(3):301-305.

21. Jeanmaire C, Danoux L, Pauly G. Glycation during human dermal intrinsic and actinic ageing: an in vivo and in vitro model study. $\mathrm{Br} J$ Dermatol. 2001;145(1):10-18.

22. Dunn JA, McCance DR, Thorpe SR, Lyons TJ, Baynes JW. Age-dependent accumulation of $\mathrm{N}$ epsilon-(carboxymethyl)lysine and $\mathrm{N}$ epsilon(carboxymethyl)hydroxylysine in human skin collagen. Biochemistry. 1991;30(5):1205-1210.

23. Gkogkolou P, Bohm M. Advanced glycation end products: key players in skin aging? Dermatoendocrinol. 2012;4(3):259-270.

24. Bogdanowicz P, Haure M-J, Ceruti I, Bessou-Touya S, Castex-Rizzi N. Results from in vitro and ex vivo skin aging models assessing the antiglycation and anti-elastase MMP-12 potential of glycylglycine oleamide. Clin Cosmet Investig Dermatol. 2016;9:143-150.

25. Bacqueville D, Douki T, Duprat L, et al. A new hair follicle-derived human epidermal model for the evaluation of sunscreen genoprotection. J Photochem Photobiol B. 2015;151:31-38.

26. Boisnic S, Branchet-Gumila MC, Le CharpentierY, Segard C. Repair of UVA-induced elastic fiber and collagen damage by $0.05 \%$ retinaldehyde cream in an ex vivo human skin model. Dermatology. 1999;199(suppl 1): 43-48.

27. Boisnic S, Branchet-Gumila MC, Merial-Kieny C, Nocera T. Efficacy of sunscreens containing pre-tocopheryl in a surviving human skin model submitted to UVA and B radiation. Skin Pharmacol Physiol. 2005;18(4):201-208.

28. Kang S, Duell EA, Fisher GJ, et al. Application of retinol to human skin in vivo induces epidermal hyperplasia and cellular retinoid binding proteins characteristic of retinoic acid but without measurable retinoic acid levels or irritation. J Invest Dermatol. 1995;105(4):549-556.

29. Ho ET, Trookman NS, Sperber BR, et al. A randomized, double-blind, controlled comparative trial of the anti-aging properties of nonprescription tri-retinol $1.1 \%$ vs. prescription tretinoin $0.025 \%$. J Drugs Dermatol. 2012;11(1):64-69.

30. Kligman AM, Grove GL, Hirose R, Leyden JJ. Topical tretinoin for photoaged skin. J Am Acad Dermatol. 1986;15(4 pt 2):836-859.
Clinical, Cosmetic and Investigational Dermatology

\section{Publish your work in this journal}

Clinical, Cosmetic and Investigational Dermatology is an international, peer-reviewed, open access, online journal that focuses on the latest clinical and experimental research in all aspects of skin disease and cosmetic interventions. This journal is included on PubMed. The manuscript management system is completely online

\section{Dovepress}

and includes a very quick and fair peer-review system, which is all easy to use. Visit http://www.dovepress.com/testimonials.php to read real quotes from published authors 